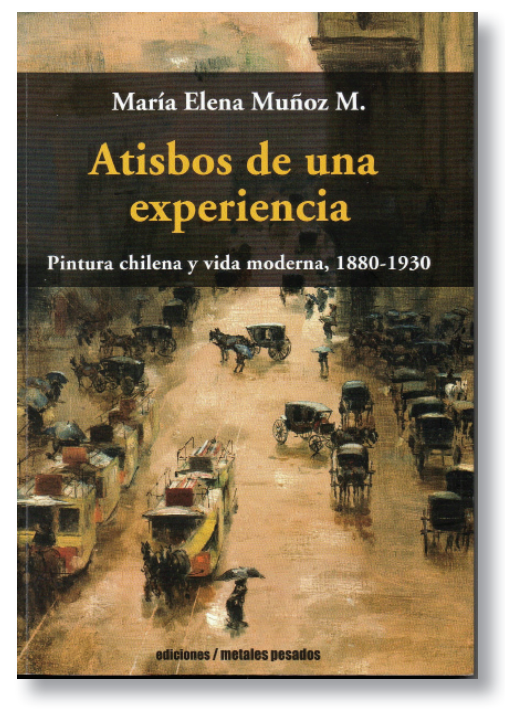

\title{
María Elena Muñoz M. \\ Atisbos de una experiencia. \\ Pintura chilena y vida moderna. 1880-1930
}

Santiago de Chile: Metales Pesados, 2014.

\section{Por Constanza A. Robles Sepúlveda Instituto de Estética - Pontificia Universidad Católica de Chile. Chile. cnrobles@uc.cl}

La modernidad es lo transitorio, lo fugitivo, lo contingente, la mitad del arte, cuya otra mitad es lo eterno y lo inmutable.

Baudelaire

Ya desde la portada -el detalle de una pintura de Ramón Subercaseaux-, la académica e investigadora María Elena Muñoz, nos deja ver su particular interés por la relación que existe entre la pintura y la modernidad hacia fines del siglo diecinueve y principios del veinte. El impulso modernizatorio que vive Chile en esa época, se ve expresado en la obra de varios pintores chilenos que ella destaca; ya sea por medio del tema de la obra, manteniendo parámetros relativamente academicistas, "El criterio para escogerlas fue que representaran, de distinto modo y con diversos énfasis, la experiencia de la vida moderna en la capital chilena. Se trata por tanto de paisajes urbanos, pero no de cualquier paisaje urbano; la premisa es que representen la ciudad, pero además la experiencia de habitarla a merced de las iniciativas modernizadoras en el ámbito de las obras civiles, la arquitectura y el y el urbanismo" (7); o en la manera de pintar sin esconder ya la pincelada, sino que, de una u otra manera, empastando la tela 
con abundante materia, "Que dieran cuenta de una sintonía con las innovaciones habidas en los lenguajes de la representación artística, simultáneas a los progresos modernizadores" (7), sobre todo, como expone la autora, en Juan Francisco González.

En los capítulos que componen el libro, Muñoz estudia acuciosamente la obra de seis artistas chilenos, a saber, Ramón Subercaseaux, Juan Francisco González, Rafael Correa, Alberto Orrego Luco, Cosme San Martín y Alfredo Helsby. La manera en que se desenvuelve el texto es analizando una o dos obras de un autor respecto a un mismo tema, o comparando dos de ellos con paisajes similares. Cabe destacar aquí, que la noción de paisaje es en el libro un concepto fundamental, sobre todo por la definición que toma Muñoz, considerándolo no sólo por su connotación ligándolo al mudo rural y económico, sino que más bien en sintonía con la palabra "país", como una manera de concebir la naturaleza que nos rodea. Pero su principal análisis tiene que ver con la extracción de información que a través de ellas se obtengan para su análisis. En este sentido, el concepto de paisaje se convierte en un elemento teóricamente fértil en tanto da pie para analizar las obras desde distintos aspectos, su vínculo con lo nacional y la idiosincrasia cultural de la época, entre otros. El paisaje se convierte en el género de la emancipación, de la naturaleza domesticada, la contención: una estetización del progreso.

Por otro lado, es importante destacar, que de las diecisiete obras analizadas, once de ellas permanecen en colecciones privadas a las que el público, lógicamente, no tiene acceso. Tres se encuentran en el Banco Central y sólo cuatro en Museos. Es por esto que además de ser relevante el trabajo en torno a las obras, lo es en igual medida, el trabajo investigativo y de archivo que realiza la académica para localizar y acceder a dichas pinturas, lo que nos permite también, como lectores, aunque sea a través de reproducciones, acceder a ellas. Cabe mencionar el que al hablar de Nevado en Providencia (1912) de Alfredo Helsby, menciona otro cuadro similar al nevado también de Helsby al que no se pudo tener acceso por encontrarse en una colección privada.

Esto convierte a "Atisbos de una experiencia" no sólo en un libro sobre pintura chilena de un determinado periodo de modernización en nuestro país, sino también en un documento de arte, un registro que apela a la carencia de una visión y acceso a las pinturas como obras de arte que debiesen encontrarse disponibles para todos, por ejemplo, en el Museo Nacional de Bellas Artes, pero que en su tiempo no fueron vistas con la calidad que el sabio ojo del tiempo nos da para valorarlas. "Acaso un prejuicio crítico y curatorial ha atentado contra su mayor difusión en colecciones públicas: se las ha considerado más documento que arte" (12). Al menos nos permite entender en parte la precariedad con que se construye y mantiene, en cuanto a adquisiciones, el museo que debiese ser el más relevante de nuestro país.

María Elena Muñoz, nos da una visión del Santiago decimonónico, y del tipo de arte que se realizaba en aquel tiempo, junto con sus clases y su idiosincrasia más profunda. Evidencia, por ejemplo, el momento en que Europa deja de ser el centro de las artes y la arquitectura, y Estados Unidos comienza a ser un referente de in- 
dustrialización, con la paulatina aparición del automóvil en desmedro de los carros de sangre. "A partir de la década del veinte, en cambio, empiezan a ser visibles otros paradigmas arquitectónicos y urbanísticos, toda vez que lo francés cede terreno a los patrones culturales norteamericanos" (9). Con esto va quedando de manifiesto el cambio hacia el continente americano que de una u otra manera nos da la posibilidad, por primera vez, de una ventaja geolocal.

Se deja entrever la pasión y el gusto de la investigadora por las obras que ha escogido, una característica que le da una fuerza particular al texto y que es generalmente obviada en la teoría y en la historia del arte, pero que no quita, sino que enriquece y potencia la lectura de las obras y la experiencia del lector, pues sus lecturas son contemporáneas y atingentes de una manera en que la teoría de arte no suele serlo. Podemos incluso asociar sus reflexiones a la modernidad tardía que deviene en la actual hipertecnologización que se vuelve permanente en el mundo entero, pero que en particular afecta a países como el nuestro, desarrollándose de una manera cada vez más voraz. A su vez, las notas de Muñoz son interesantes y pertinentes pues no están ligadas sólo al arte sino que también a la arquitectura, a la política y la cultura de la época, lo que hace su análisis aún más relevante al relacionarse con una construcción de ciudad en su acepción más compleja y no sólo en su visualidad. "Tal periodo corresponde al momento en que algunas ciudades latinoamericanas lograron subirse a carro del progreso de tal modo que pudieron exhibir cambios importantes en sus fisonomías, así como notorias transformaciones sociales y culturales" (7).

De la misma manera nos habla de temas esenciales para la modernidad, los que son reconocidos desde un principio por Charles Baudelaire -el poeta de la modernidad por antonomasia- y retomados después (tardíamente) por críticos chilenos como Antonio Romera. La modernidad asociada al concepto de mal tiempo que expone Muñoz en su libro, como una cruzada citadina que se ve esperanzada por la llegada del alumbrado eléctrico y la aparición del hombre de mundo, que plantea Baudelaire. "La mayoría de las veces se trata de escenas que transcurren bajo un clima adverso, un cielo gris y una atmósfera húmeda, como si el reflejo de esas condiciones climáticas metaforizara la pesadumbre que inundaba a esa misma experiencia donde todo parecía desvanecerse en el aire" (11). El mal tiempo, como se plantea aquí, es un tema muy presente durante la modernidad. Sin ir más lejos, el mismo Baudelaire ha escrito numerosos poemas sobre ella (Cisne, A una transeúnte y Bruma y lluvia, por citar algunos ejemplos de su libro "Las flores del mal") y artistas como Gustave Caillibotte y Camille Pissarro son ejemplos canónicos de la expresión de la modernidad en este sentido dando vistas a la ciudad y a la presencia de este mal tiempo como expresión del sentir interior de la creciente multitud que se gesta en la época. Este es otro de los conceptos que nos introduce Baudelaire en "El Pintor de la vida moderna"1 y que se ve

1 Baudelaire, Charles. Salones y otros escritos sobre arte. Madrid: A. Machado Libros, 2005. 
subyace en este libro: "la multitud es su dominio, como el aire es el del pájaro, como el agua el del pez. Su pasión y su profesión es adherirse a la multitud. Para el perfecto paseante, para el observador apasionado, es un inmenso goce el elegir domicilio entre el número en lo ondeante, en el movimiento, en lo fugitivo y lo infinito". (Baudelaire, 358). Muchas cosas se desvanecían o eran desvanecidas, por el progresivo plan de higienización, como estado de modernidad última, pero también como forma de embellecer la ciudad y depurarla de aquellos elementos que pudiesen asociarla a la barbarie, como cita Muñoz, en oposición a la búsqueda de civilización.

En los diez capítulos, donde se despliega el análisis, vemos cómo salen a relucir los distintos ejes temáticos que conducen el libro. En el primer capítulo, encontramos dos obras de Ramón Subercaseaux: Plaza de la compañía, 1884 y Plaza de Armas, 1888. En la primera, destaca la condición invernal y devenir de la multitud hecho que relaciona al impresionismo: pulso de modernización y experiencia anímica de la modernidad, mientras que en la segunda, se da cuenta de la iluminación eléctrica "la pintura remite a una cierta 'irrealidad' como consecuencia de la alteración de la escala y de la iluminación artificial del edificio principal” (33). En el sexto capítulo encontramos una comparación entre Plaza Italia (Baquedano), 1931 de Ramón Subercaseaux y Plaza Italia (circa 1925) de Juan Francisco González, comparación que deja en evidencia el origen de González, desde la chimba, el lado otro, el lado bárbaro, desinteresado de la vida moderna.

No así en el análisis que hace posteriormente de Alameda (circa 1890) de Juan Francisco González, imaginario inaugural de la modernidad pictórica local. Se aprecian hombres, acaso oficinistas pertenecientes a la emergente clase media o estudiantes que se acercan a la casa central de la Universidad de Chile. Hay una figura que mira al espectador, la autora especula que puede ser el mismo artista, obra que complementa con Panorama de Santiago (circa 1910), del mismo artista, donde se hace presente, casi de manera exagerada, la técnica a través del uso en exceso de pintura, evidenciando la pincelada y la pasta oleosa que compone la obra. Aquí, la modernidad está en la materia y no en lo representado.

En los últimos capítulos del libro, Muñoz vuelve a Ramón Subercaseaux con cuatro obras, dos tituladas Monumento a O'Higgins (circa 1928) y (circa 1920), donde destaca la imagen de la mujer que pasa frente al espectador de espaldas al monumento como alguien que irrumpe sin querer en una fotografía; y en la segunda, la autora plantea la idea de que "es en la organización del espacio perspéctico (la ilusión tridimensional) que se trama un devenir histórico: lo más lejano es lo más anterior en el tiempo, y en la medida en que nos acercamos al primer plano nos acercamos a un tiempo nuevo" (159). Por otro lado, vemos dos obras situadas en Calle Morandé, ambas de 1934. Aquí se muestra una de las primeras evidencias de que, por la preponderancia del automóvil, se comienza a alejar la mirada de Europa y se acerca a Norteamérica, nación pragmáticamente más eficiente. Subercaseaux fue el pintor de la modernidad en Santiago de la élite ilustrada, donde se ve que una de las premisas vitales para la 
escritora es la ciudad como escenario de la vida pública (34). Aquí es interesante detenerse en la relación que se puede establecer entre fotografía y pintura, siendo la fotografía un punto de inflexión en la manera de pintar, donde el retrato como se conocía hasta entonces pasa a segundo plano y desafía a la pintura a ser más que el reflejo representativo de lo real, esa ya es tarea de la máquina fotográfica. La pintura toma las virtudes de la fotografía y la incorpora elaborando nuevos encuadres, hasta el momento impensados en el marco de la tela.

Para terminar, cabe destacar, que si bien el libro versa sobre la pintura en Santiago del Siglo diecinueve, nos permite analizar distintos aspectos de la realidad contemporánea. Mediante las obras hemos podido ver mucho de la historia del desarrollo de Santiago, el que ha cambiado mucho entrado el siglo veintiuno, habiendo descuidado hoy, barrios patrimoniales muy importantes como el Barrio Yungay, concentrando sus esfuerzos en la creación de malls y estructuras similares a lo largo del país. Nos queda claro que "persigue abrir vetas para explorar vínculos, complicidades, correspondencias y contradicciones entre ellas y sus entornos, entre sus entornos y los nuestros" (14).

"Atisbos de una experiencia", hace un recorrido por la pintura chilena y la vida moderna de fines del siglo diecinueve y principios del veinte. Afortunadamente, nos permite ir más allá de la pintura, nos acerca a la fotografía, a la instantánea y a lo fugaz, al mismo tiempo que nos obliga a releer nuestra historia y entender la contemporaneidad desde nuevas perspectivas. Es un libro de historia del arte, pero también un libro sobre la ciudad de Santiago y cómo se constituye el eje central de nuestra vida moderna. María Elena Muñoz, traza un recorrido, se aventura en una novela pictórica de hombres con sombrero de copa y mujeres en pomposos vestidos paseándose por la Alameda, pero donde ellos no son los protagonistas, sino parte escenográfica del verdadero centro de la época: la ciudad. Una ciudad que nos interpela, ya no desde sus cambios finiseculares, sino de cómo nos desenvolvemos en esa misma ciudad, hoy casi irreconocible. Sin que el progreso sea algo negativo, el poder situarnos en lo que fue y continúa siendo la ciudad de Santiago, mirando de otra manera, los parques y fachadas de edificios con los que nos topamos a diario. La académica, desde su interés investigativo tan particular, nos permite leer un libro mucho más actual de lo que aparenta, hecho que en estos tiempos, se ha convertido en un bien escaso. 